\title{
Revista Brasileira de Enfermagem REBEn \\ Teste do reflexo vermelho: forma de prevenção à cegueira na infância
}

\author{
Red reflex: prevention way to blindness in childhood \\ Test del reflejo rojo: forma de prevención de la ceguera en la niñez
}

\section{Adriana Sousa Carvalho de Aguiar}

Aluna do Curso de Graduação em Enfermagem da Universidade Federal do Ceará, Fortaleza, CE. Bolsista PIBIC - CNPq. Membro do Projeto Saúde Ocular. adrianaufc@gmail.com

\section{Maria Vera Lúcia Moreira Leitão Cardoso}

Enfermeira, Doutora Professora Adjunto do Departamento de Enfermagem da

Universidade Federal do Ceará, Fortaleza, CE. Membro do Projeto Saúde Ocular, Coordenadora da Área Saúde Ocular da

Criança. cardoso@ufc.br.

\section{Ingrid Martins Leite Lúcio}

Enfermeira. Mestre. Professora Substituta do Departamento de Enfermagem da Universidade Federal do Ceará, Fortaleza,

CE. Doutoranda do Programa de PósGraduação em Enfermagem. Bolsista CAPES. Membro do Projeto Saúde Ocular. ingrid lucio@yahoo.com.br

Trabalho desenvolvido no Projeto Saúde Ocular/CNPq. Prêmio Zaira Cintra Vidal, $1^{\circ}$ lugar no $58^{\circ}$ Congresso Brasileiro de Enfermagem.

Submissão: 09/01/2006 Aprovação: 03/07/2007

\section{RESUMO}

Estudo exploratório, quantitativo, que objetivou investigar o resultado e as gradações de cor do teste do reflexo vermelho em recém-nascidos (RN). Amostra composta de $180 \mathrm{RN}$ de uma maternidade em Fortaleza-CE. Destes, 156 tiveram resultado "não alterado" e 24 "suspeito". Quanto ao aspecto do reflexo, em $144 \mathrm{RN}$ a coloração foi a mesma nos dois olhos, enquanto em 35 apresentou-se vermelha, em 33, laranja avermelhada, em 46, alaranjada, em 24, amarelo claro, em 6 amarela com manchas esbranquiçadas centrais. Dos casos suspeitos, o reflexo mostrou-se amarelo claro com manchas esbranquiçadas ou rajadas. 0 enfermeiro treinado é um dos profissionais competentes para realizar o teste, podendo desempenhar importante papel na Unidade Neonatal com ações de prevenção de alterações oculares na infância.

Descritores: Saúde ocular; Enfermagem neonatal; Cegueira.

\section{ABSTRACT}

This study had as objective to investigate the result and the colour gradation of red reflex test in newborns (NB). It is a exploratory, quantitative study and the sample was 180 NB from maternity ward in Fortaleza-CE. From this, 156 showed result "no altered" and 24 "suspect". About the aspect of red reflex, 144 NB showed the same coloration in the two eyes, in 35 of this, the colour was red, in 33, orange reddish, in 46 orange colour, in 24 light yellow, in 6 yellow with whitish stains central. Of the suspect cases, the reflex was light yellow with whitish stains with lines. The nurse trained to accomplish the red reflex test can have important role at Neonatal Unit with actions about the prevention of ocular alterations in the childhood.

Descriptors: Eye health; Neonatal Nursing; Blindness.

\section{RESUMEN}

Estudio exploratorio, cuantitativo, que tuvo como objeto investigar el resultado y las gradaciones de color del test del reflejo rojo en recién nacidos (RN). La muestra constó de $180 \mathrm{RN}$ de una maternidad en Fortaleza-CE. De estes, 156 presentaron resultado "no alterado" y 24 "sospechoso". Cuanto al aspecto del reflejo, $144 \mathrm{RN}$ presentaron la misma coloración en los dos ojos, de estes 35 presentó rojo, 33 naranja rojizo, 46 anaranjado, 24 amarillo claro, 6 amarillo con manchas blanquecinas centrales. De los casos sospechosos, el reflejo presentó amarillo claro con manchas blanquecinas con rayas. El enfermero entrenado para realizar el test del reflejo rojo puede desempeñar importante papel en la Unidad Neonatal con acciones de prevención de alteraciones oculares en la niñez.

Descriptores: Salud ocular; Enfermería neonatal; Ceguera.

Aguiar ASC, Cardoso MVLML, Lúcio IML. Teste do reflexo vermelho: forma de prevenção à ceguiera. Rev Bras Enferm 2007 set-out; 60(5): 541-5.

\section{INTRODUÇÃO}

A visão é uma das principais fontes de estímulo ao desenvolvimento físico e cognitivo a partir dos primeiros momentos de vida, e sua importância individual e coletiva pode ser avaliada. Para entendermos o significado da visão para os seres humanos, vale ressaltar que os gestos e condutas sociais são aprendidos pelo feedback visual. Conforme sabemos, a cegueira implica uma série de conseqüências sociais e econômicas para o indivíduo e a sociedade. Portanto, constitui um sério problema de saúde pública, ainda mais agravado quando consideramos que a maioria desses casos são preveníveis ou tratáveis pela adoção de medidas simples.

Segundo revelado por estudos nessa área, mesmo com o conhecimento e a tecnologia atual, muitas das alterações visuais ocorridas na infância são identificadas tardiamente, quando, dependendo do 
diagnóstico, pouco efetiva poderá vir a ser a intervenção(1). Contudo, em países em desenvolvimento, 30 a 72\% da cegueira infantil são evitáveis, 9 a 58\% são preveníveis e 14 a 31\% são tratáveis(2).

Nesse contexto, as estimativas mundiais a respeito da prevalência e incidência da cegueira evidenciam a necessidade de planejar e de desenvolver estratégias voltadas para a promoção da saúde ocular e detecção precoce de alterações visuais.

De acordo com a literatura, a cegueira em crianças está relacionada com o desenvolvimento socioeconômico: $75 \%$ das crianças cegas no mundo vivem em países em desenvolvimento, sendo a prevalência nestes países de 15/10.000, ou seja, cinco vezes maior que a encontrada nos países desenvolvidos ${ }^{(3)}$.

Entre as doenças oculares, uma das principais responsáveis pela perda visual (parcial ou total) em crianças é a retinopatia da prematuridade (RP). Definida como um distúrbio vasoproliferativo de origem multifatorial, acomete a retina do recém-nascido prematuro de baixo peso e seu desenvolvimento está relacionado ao uso de oxigenoterapia. Embora possua estágios variáveis, desde efeitos mínimos nos vasos da retina, até o seu descolamento total, a RP tem cura espontânea em $90 \%$ dos casos. Todavia, $6 \%$ chegam a necessitar de tratamento cirúrgico(4).

Outro achado importante na população e no recém-nascido é a catarata congênita, decorrente de alterações na formação do cristalino, cujas causas podem ser as seguintes: infecções intra-uterinas (rubéola, toxoplasmose, citomegalovírus e sífilis), traumatismo, prematuridade, hereditariedade, uso de medicamentos, síndromes genéticas, malformaç̧̃es oculares congênitas, ou origem idiopática. Considerada uma causa freqüente de cegueira evitável na infância, esta patologia pode ser identificada por meio do teste do reflexo vermelho, focalizando-se a opacidade, que varia desde tênue até suficientemente densa para dar o aspecto de leucocoria, pupila branca ${ }^{(5)}$.

De forma geral, existe mais preocupação com as alterações visuais que afetam a criança, tanto na vida intra-uterina como após o nascimento (nos primeiros anos de vida). Isso ocorre porque este é o período de formação do desenvolvimento orgânico e da estabilidade funcional da visão, e, portanto, de maior vulnerabilidade à agressão por diferentes agentes etiológicos ${ }^{(6)}$.

Por esta razão, são necessários o cuidado e a adoção de intervenções multiprofissionais dirigidas à prevenção de alterações visuais a partir do prénatal e, por conseguinte, no período pós e neonatal. A finalidade é rastrear fatores congênitos, distúrbios neurológicos e infecções maternas, como a rubéola, a sífilis e a toxoplasmose, passíveis de comprometer a visão do recém-nascido, além de prevenir fatores adquiridos causados por determinadas terapêuticas, como a oxigenoterapia, a fototerapia e transfusões sangüíneas ${ }^{(7,8)}$.

Como mostra o dia-a-dia, as condições de prematuridade e baixo peso ao nascer, bebês com hipóxia grave e antecedentes maternos de doenças infecciosas gestacionais (sífilis, rubéola, vulvovaginites, infecções urinárias) constituem fatores de risco para complicações visuais geralmente associadas a outros fatores. Além disso, alguns tratamentos como fototerapia e oxigenoterapia necessários para atender às funções vitais do recém-nascido, quando inadequadamente monitorizados e controlados, podem refletir em iatrogenias no sistema visual(9).

Uma das formas de se detectar alterações visuais, que podem passar despercebidas pela equipe de saúde responsável pelos cuidados ao recémnascido, é a prática do teste do reflexo vermelho ou teste do reflexo de Bruckner, também conhecido por teste do olhinho.

Mas o objetivo desse teste não é visualizar as estruturas da retina (vasos, disco óptico e mácula), porquanto isto é feito pelo exame da fundoscopia, e sim avaliar se existe algum obstáculo à chegada da luz até ela (opacidade de córnea, catarata, hemorragias vítreas, etc. ou por doenças dela própria, como tumores (por exemplo, retinoblastoma) ${ }^{(10)}$. Apesar de sua utilidade, atualmente, só nos estados do Rio de Janeiro e São Paulo o teste do reflexo vermelho é rotina obrigatória, por lei, nas salas de parto.

O TRV é um exame simples e rápido. Quando a luz estiver diretamente alinhada ao longo do eixo visual de uma pupila dilatada, o espaço pupilar aparecerá como um brilho homogêneo de cor vermelho-alaranjada. Este é o chamado reflexo vermelho, ou seja, é o reflexo da cor do fundo do olho, através do meio ocular, aquoso e córnea ${ }^{(11)}$. Porém, conforme observamos na nossa prática, o termo referido na literatura como "reflexo vermelho" nem sempre corresponde fielmente ao que é achado ao se realizar o teste, pois aparecem várias outras tonalidades não necessariamente vermelhas, as quais podem ser consideradas sem alteração. Ressalta-se que a coloração do reflexo observada no exame está relacionada a algumas variáveis, como estágio de desenvolvimento da retina, idade gestacional do recém nascido, uso de terapêuticas como oxigênio, iluminação do ambiente e posicionamento do RN. Este pode interferir no alinhamento do seu eixo visual.

Realizado com o auxílio de um oftalmoscópio direto que emite uma luz que irá transpor os meios transparentes do olho refletindo a coloração da retina, a principal importância desse teste consiste na detecção precoce de doenças, como a catarata congênita e a leucocoria, que comprometem 0 eixo visual. Como sugerido por estudiosos, essa avaliação do reflexo vermelho deveria se constituir num exame de rotina no período neonatal, pois permite avaliar a opacidade do cristalino ${ }^{(12)}$.

A despeito disso, no Brasil, a literatura em enfermagem sobre 0 assunto é escassa. Ademais, as ações em enfermagem na área de oftalmologia são tímidas, sendo mais efetivas em países desenvolvidos, como os Estados Unidos e a Inglaterra( ${ }^{(9)}$.

Particularizando nossa experiência no Brasil, especificamente no Ceará, ressaltamos a existência do Projeto de Pesquisa Saúde Ocular, o qual faz parte do Departamento de Enfermagem da UFC. Financiado pelo CNPq desde 1994, trabalha com cinco grandes temáticas, entre elas, a Saúde Ocular da Criança, na qual se enquadra esse estudo. Esta vem desenvolvendo ações que envolvem desde a avaliação visual, incluindo 0 exame das estruturas oftálmicas externas, o teste do reflexo vermelho até a estimulação visual e encaminhamento para oftalmologista. Todo esse processo em andamento desde $1995 \mathrm{vem}$, a cada dia, fortalecendo as ações dos integrantes do projeto, favorecendo o aprofundamento de conhecimentos mediante estratégias destinadas a contribuir para a prevenção da cegueira no Brasil, e mais especificamente no Ceará.

Diante da relevância dos estudos na área da saúde ocular, é preciso despertar para a necessidade de inserir na rotina de cuidados a avaliação visual pelo enfermeiro. Dessa forma ele estará somando esforços com outros profissionais para a prevenção e redução da cegueira infantil.

Como mencionado, esta pesquisa objetivou investigar o resultado do teste do reflexo vermelho em recém-nascidos internados na Unidade Neonatal e Sistema de Alojamento Conjunto e averiguar quais as gradações de cores mais comuns do reflexo vermelho.

\section{METODOLOGIA}

Estudo descritivo-exploratório, quantitativo, desenvolvido nas unidades de internação neonatal, envolvendo também o alojamento conjunto e a enfermaria "mãe-canguru", em uma maternidade pública de grande porte, centro de referência para o Município de Fortaleza e para o Estado do Ceará.

A população foi constituída por recém-nascidos internados nos setores citados. Participaram da amostra 180 recém-nascidos independentemente de peso, idade gestacional e condições ao nascimento, estando fora do estado crítico de saúde. 0 valor da amostra está baseado no número de partos mensais da maternidade onde se realizou o estudo, em torno de 600 partos/mês. Portanto, trabalhamos com 30\% dessa população.

Utilizamos como meio de coleta de dados um formulário estruturado, composto por duas partes: história materna e neonatal e exame ocular do recém-nascido.

Além desse instrumento, utilizamos um gradiente de cores para identificar 
qual a coloração mais comum do reflexo observado. Esse gradiente de cores foi criado a partir da nossa prática do teste do reflexo vermelho, quando detectamos certa variância das cores que nos remeteu ao resultado duvidoso do teste no início de 2005.

O formulário estruturado abordou vários aspectos, entre eles: dados relacionados a condições gestacionais e de nascimento da criança; dados do pré-natal, referentes à mãe, como história de infecção; avaliação das estruturas oculares externas; investigação das respostas visuais básicas do RN que envolvem fixação e seguimento visual; e a realização do teste do reflexo vermelho.

Para o preenchimento dos dados do formulário recorremos aos registros encontrados nos prontuários, às vezes com a colaboração dos pais, e por meio do exame propriamente dito, conduzido na mesma unidade na qual o $\mathrm{RN}$ se encontrava.

Para avaliação do reflexo vermelho, adquirimos um oftalmoscópio direto via Projeto Saúde Ocular/ UFC/ CNPq. Em continuidade à pesquisa, fizemos levantamento de literaturas que contemplassem 0 assunto e a técnica de avaliação do reflexo vermelho. Também obtivemos informações feitas pela oftalmologista colaboradora desse estudo, que se comprometeu a avaliar os recém-nascidos com resultados suspeitos ou alterados do reflexo vermelho.

A coleta dos dados verificou-se no período de novembro de 2005 a março de 2006. Mantivemos contato com a mãe ou responsável pelo RN para informar quanto aos objetivos da pesquisa, benefícios e inexistência de riscos e, então, pedir o consentimento para a participação do RN.

Com vistas à realização do exame ocular, utilizamos os métodos propedêuticos de inspeção e palpação para o exame das estruturas oculares externas, com uma lanterna para a avaliação do reflexo pupilar e um oftalmoscópio direto para realização do teste do reflexo vermelho.

A atividade visual, que envolveu a sensibilidade ao contraste, a fixação e seguimento visual fugazes, foi observada por meio de artefatos com figuras em preto e branco, com listras verticais alternadas ou círculos concêntricos.

Para a realização do teste do reflexo vermelho alguns aspectos importantes devem ser observados: a) ambiente em penumbra para proporcionar dilatação fisiológica das pupilas; b) uso adequado das lentes do oftalmoscópio de 0 para +2, ou +5, conforme a correção óptica do examinador; c) direcionamento do foco luminoso para ambos os olhos da criança simultaneamente, numa distância entre 40 e $50 \mathrm{~cm}$; d) observação da presença do reflexo que normalmente é vermelho alaranjado, ao incidir a luz através da pupila, de modo que fique alinhada ao eixo visual.

Os dados obtidos foram descritos e apresentados em tabelas e sua análise respaldou-se na literatura pertinente ao tema. Em todas as etapas os preceitos éticos referentes à pesquisa com seres humanos foram respeitados, conforme a Resolução 196, de 10 de outubro de 1996, do Conselho Nacional de Saúde. Após a aprovação pelo Comitê de Ética do Complexo Hospitalar da Universidade Federal do Ceará, as mães ou responsáveis pelos RN foram orientadas quanto aos objetivos e finalidades da pesquisa e assinaram um termo de consentimento autorizando a avaliação visual do filho. Foi-lhes garantido o total anonimato, assim como a possibilidade da desistência de participar do estudo caso quisessem, sem prejuízo do tratamento do seu filho.

\section{RESULTADOS E DISCUSSÃO}

Ao conduzir a avaliação visual, é necessário conhecer um pouco da história pré-natal, pois fatores importantes podem ser identificados e guardar relação com alterações apresentadas pelo RN. Por isso, inicialmente falaremos da caracterização das mães, posteriormente dos recém-nascidos, e a seguir sobre os dados do exame ocular das estruturas externas, 0 seguimento visual e os resultados do teste do reflexo vermelho.

Quanto às variáveis, recorremos aos prontuários dos recém-nascidose complementamos com informações das mães.
Os dados relacionados à história materna envolveram aspectos da história pregressa da mãe, relacionados às condições gestacionais, ao parto, infecções durante o pré-natal, como rubéola, toxoplasmose e sífilis. A história oftalmológica foi investigada quanto aos casos de erro de refração, catarata e glaucoma, familiar ou pessoal.

Nesta etapa investigamos 177 histórias maternas. Em virtude de três partos gemelares, este total foi diferente do de recém-nascidos. Para as mães desses bebês o perfil foi o seguinte: a idade materna variou entre 14 e 46 anos e 60 (33,3\%) mães tinham idade inferior a 20 anos. Além disso, $40(22,2 \%)$ delas apresentaram história de aborto anterior e $35(19,4 \%)$ tinham história de vícios (etilismo ou tabagismo). Ainda conforme os dados, $49(27,2 \%)$ apresentaram infecção urinária durante o pré-natal, 52 (28,8\%) desenvolveram Doença Hipertensiva Específica da Gravidez (DHEG), 12 $(6,6 \%)$ hemorragia no $1^{\circ}$ ou $2^{\circ}$ trimestre da gestação e $2(1,1 \%)$ eram portadoras do Papiloma Vírus Humano (HPV), manifestado por condiloma genital. Também de acordo com os dados, 4 (2,2\%) das mães tinham sífilis, 2 (1,1\%) apresentaram IgG (anticorpos) para rubéola e citomegalovírus e outras $2(1,15)$ tinham herpes genital. Outras mães, $11(6,1 \%)$, eram portadoras de diabetes gestacional, $70(38,8 \%)$ tinham história familiar ou pessoal de erros de refração e $62(34,0 \%)$ de catarata.

Como percebemos, os fatores relevantes na história materna mostraram relação com o parto prematuro, e a prematuridade é um fator-chave para se investigar alterações visuais em recém-nascidos. Segundo referido na literatura, a sífilis pode levar ao trabalho de parto e nascimento prematuros. Se a infecção for congênita, pode causar anomalias oculares. Entre outras complicações, a rubéola pode provocar catarata e glaucoma; a infecção por citomegalovírus pode levar à cegueira; a infecção pelo vírus herpes tipo 2 causa infecção nos olhos e mucosas e as infecções geniturinárias, que podem ter como agente etiológico a Neisseria gonorrheae, transmitida pelo parto normal, ocasionam oftalmia neonatal ${ }^{(13,14)}$.

Neste estudo consideramos importante mencionar as intercorrências apresentadas pela mãe no pré-natal, como: diabetes gestacional, DHEG, hemorragias, infecção urinária, HPV e tabagismo. A maioria destas intercorrências contribuem para o parto prematuro. Este pode levar a uma imaturidade geral do RN e ocasionar disfunção em qualquer órgão ou sistema, inclusive o visual.

Quanto às características da história neonatal, do total dos 180 recémnascidos, $78(43,3 \%)$ nasceram por parto normal, $98(54,4 \%)$ por cesariana e 4 (2,25\%) por fórceps. Ademais, 94 recém-nascidos (52,2\%) nasceram com idade gestacional inferior a 37 semanas, portanto, considerados recémnascidos pré-termos, e os outros $86(47,7 \%)$ nasceram a termo (entre 37 e 42 semanas de gestação). 0 peso dos recém-nascidos variou de 715 a 4.900 gramas, e $89(49,4 \%)$ deles foram classificados como de baixo peso (inferior a 2.500 gramas). Ao correlacionarmos peso e idade gestacional (IG) verificamos que $46(25,5 \%)$ recém-nascidos foram considerados PIG - Pequenos para a Idade Gestacional; 118 (65,5\%) AIG - Adequados para a Idade Gestacional e 16 (8,8\%) GIG - Grandes para a Idade Gestacional. Os escores do APGAR variaram no primeiro minuto de 2 a 10 e no quinto minuto de 4 a 10. Diante disto, 76 (42,2\%) bebês foram submetidos a fototerapia. Como sabemos, as modalidades de oxigenoterapia utilizadas englobam o oxigênio por hood ou capacete, o CPAP (nasal) - pressão positiva contínua nas vias aéreas, e a ventilação mecânica, de maneira isolada ou com associações, conforme a evolução clínica do recém-nascido. A maioria dos recém-nascidos necessitou de oxigenoterapia, no total de 139 (72,2\%). Destes, 60 (46,1\%) utilizaram apenas o oxi-hood; 41 (31,5\%) utilizaram o hood e o CPAP; 22 (16,9\%) o hood, o CPAP e a ventilação mecânica (VM) e 7 (5,3\%) o hood e a VM. Dos recém-nascidos, 22 $(12,2 \%)$ fizeram transfusão sangüínea.

Com relação às características da história neonatal, segundo observamos, uma porcentagem significativa dos RN tinha idade gestacional inferior a 37 semanas e apresentou baixo peso ao nascimento. Esses constituem fatores relevantes ao se investigar alterações visuais em RN porque se encontram 
fortemente relacionados à retinopatia da prematuridade. Além desses fatores, a administração e monitorização do oxigênio devem ser cuidadosas a fim de evitar administração desnecessária ou em altas concentrações, que podem produzir efeitos tóxicos, inclusive ao sistema visual.

Quanto à avaliação ocular externa, esta é simples e pode ser realizada durante o exame físico, em uma consulta de triagem, em ambulatórios, ou ainda em um momento de interação com o recém-nascido. Contudo, algumas estruturas devem ser observadas, como pálpebras, esclera, conjuntiva e córnea. Recomenda-se investigar presença de edema, secreção purulenta, sinais de catarata, glaucoma congênito e reação pupilar à luz. Na tabela a seguir constam os resultados quanto ao exame ocular das estruturas externas.

Segundo constatamos, 111 (61,7\%) dos 180 bebês estudados apresentaram alguma alteração ao exame ocular externo, destacando-se: 29 (16,1\%) edema de pálpebra, 21 (11,7\%) secreção ocular, 18 (10,0\%) estrabismo, $16(8,9 \%)$ pontos hemorrágicos na esclera e, em menor freqüência, 12 (6,7\%) hiperemia palpebral, 10 (5,6\%) icterícia e 5 (2,8\%) dobras epicânticas.

A literatura aponta como alterações comuns em RN o edema nas pálpebras e pontos hemorrágicos na esclera em virtude da compressão dos vasos sangüíneos no momento do trabalho de parto. Identificamos também o estrabismo, decorrente da imaturidade da musculatura ocular, alteração esta considerada fisiológica até seis meses de idade; e a esclerótica amarela, conseqüência da hiperbilirrubinemia (icterícia). Ressaltamos que a literatura considera o estrabismo fisiológico até os seis meses de vida, porém devemos atentar para os fatores de risco associados ao aparecimento de distúrbios visuais, podendo, às vezes, mesmo antes dos seis meses, a criança desenvolver estrabismo patológico, o qual é diagnosticado e tratado pelo oftalmologista.

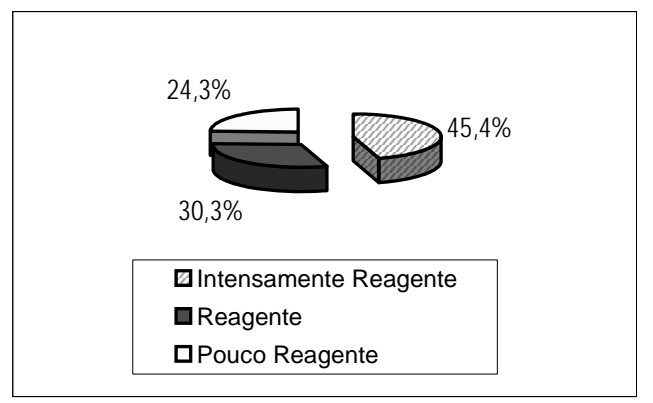

Figura 1. Distribuição das respostas dos recémnascidos ao seguimento de objetos no campo visual.

Tabela 1. Distribuição do número de recém-nascidos segundo as alterações oculares externas. Novembro de 2005 a março de 2006.

\begin{tabular}{lcc}
\hline Alterações Oculares & $\mathbf{N}^{0}$ de RN & $\%$ \\
\hline Edema Palpebral & 29 & 16,1 \\
Secreção Ocular & 21 & 11,7 \\
Estrabismo & 18 & 10,0 \\
Pontos Hemorrágicos & 16 & 8,9 \\
Hiperemia Palpebral & 12 & 6,7 \\
Icterícia & 10 & 5,6 \\
Dobras Epicânticas & 5 & 2,8 \\
Sem Alteração & 69 & 38,3 \\
\hline Total & $\mathbf{1 8 0}$ & $\mathbf{1 0 0 , 0}$ \\
\hline
\end{tabular}

De acordo com a literatura, o recém-nascido possui um campo visual restrito. Assim, a melhor distância para ele enxergar fica entre 20 e $35 \mathrm{~cm}$. Além disso, ele tem preferência por grandes contrastes em vez de objetos de cores fortes. Na figura 1, expomos os resultados das respostas do recém-nascido ao seguimento de objetos no campo visual. Com relação ao seguimento visual, apenas 152 recém-nascidos o realizaram, e 69 (45,4\%) destes apresentaram resposta classificada como intensamente reagente (olha o objeto, fixa e o acompanha), 46 (30,3\%) mostraram-se reagentes (olha o objeto, fixa, mas não o acompanha quando movimentado dentro de seu campo visual), e $37(24,3 \%)$ tiveram resposta visual pouco reagente (olha o objeto, mas não o fixa por muito tempo).

A avaliação da resposta do RN ao seguimento de objetos no seu campo visual mostrou que a maioria deles apresentou resposta classificada como intensamente reagente. Desse modo evidenciamos o interesse dos RN por um estímulo visual.

Ao realizar o teste do reflexo vermelho, conforme observamos, dos 180 recém-nascidos, 156 (86,7\%) apresentaram reflexo não alterado, ou seja, cor que varia do laranja ao vermelho. Dos recém-nascidos com reflexo suspeito, 24 (13,3\%), além da relevância das suas histórias de vida e condições de saúde, tiveram a coloração do reflexo fora do padrão, e a maioria apresentou o reflexo mais para o amarelo, com manchas esbranquiçadas ao centro, ou presença de "rajadas". Estes foram encaminhados para avaliação pelo oftalmologista.

A figura 2 ilustra o resultado do teste do reflexo vermelho realizado em recém-nascidos.

Com relação à gradação de cores do TRV, 144 (80,0\%) tinham a mesma coloração em ambos os olhos, dos quais 35 (19,4\%) apresentaram o vermelho (variando de claro a escuro); 33 (18,3\%) laranja avermelhado;

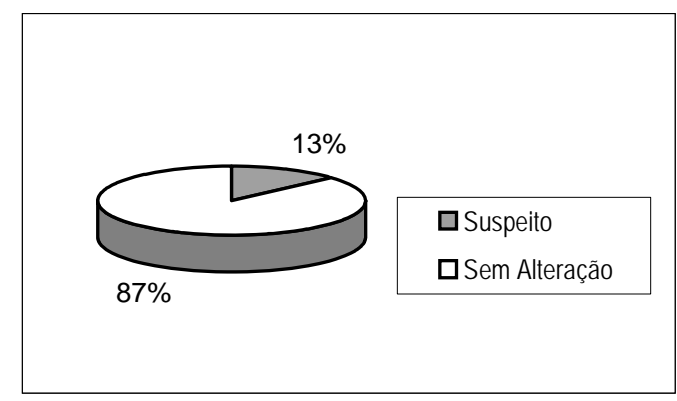

Figura 2. Distribuição do resultado do TRV observado em recém-nascidos. Novembro de 2005 - março de 2006.

Tabela 2. Distribuição com relação à gradação de cores no TRV. Novembro de 2005 - março de 2006.

\begin{tabular}{lccc}
\hline \multirow{2}{*}{ Gradação de cores do TRV } & \multicolumn{3}{c}{ N $^{\circ}$ de RN } \\
\cline { 2 - 4 } & OD & OE & Ambos \\
\hline Vermelho & 15 & 4 & 35 \\
Laranja Avermelhado & 4 & 12 & 33 \\
Alaranjado & 13 & 12 & 46 \\
Amarelo Claro & 3 & 6 & 24 \\
Amarelo c/ Mancha esbranquiçada & 1 & 2 & 6 \\
\hline Total & $\mathbf{3 6}$ & $\mathbf{3 6}$ & 144 \\
\hline
\end{tabular}


46 (25,5\%) alaranjado; 24 (13,4\%) amarelo claro e 6 (3,3\%) amarelo com discreta mancha esbranquiçada ao centro. Os demais recém-nascidos (36; 20\%) mostraram diferenças quanto à intensidade ou ao tipo de cor do TRV, ora no olho direito, ora no olho esquerdo. A tabela a seguir mostra as gradações de cores do TRV.

No referente ao TRV, conforme observamos, 24 (13,3\%) obtiveram resultado suspeito. Porém, ao analisar depois os prontuários, notamos que em 8 desses bebês foi realizada a fundoscopia pelo oftalmologista, com conseqüente alteração do resultado e definição do diagnóstico: em 5 deles, retinopatia da prematuridade; em 2, retina avascular; e em 10 achado mostrou pontos hemorrágicos na retina. Os demais RN com resultado suspeito do reflexo vermelho foram encaminhados para avaliação pela oftalmologista colaboradora deste estudo.

Como podemos observar, a maioria dos RN, 156 (86,7\%), apresentaram reflexo vermelho normal. Com relação à intensidade ou ao tipo de cor do reflexo vermelho, observaram-se diferenças, ora no olho direito, ora no olho esquerdo. Devido a essa variância de cores e à experiência em estudos anteriores, percebemos a necessidade de registrar quais as gradações de cores mais comuns, para, assim, embasar melhor a avaliação do teste. Notamos, então, a predominância da coloração vermelha, alaranjada e amarelo claro, esta última em decorrência da vascularização incompleta da retina em prematuros.

Em estudo anterior ${ }^{(15)}$, no qual foram examinados 180 recém-nascidos, obtiveram-se os seguintes resultados: $5 \%$ (9) apresentaram reflexo vermelho alterado. Diante disto, foram avaliados pelo oftalmologista para confirmação do achado, esclarecimento diagnóstico e tratamento imediato, já que a permanência da causa poderá acarretar comprometimento, algumas vezes definitivo e importante da acuidade visual. Como impressões diagnósticas mencionamos: seis recém-nascidos com retinopatia e um recém-nascido com leucocoria (pupila branca).

\section{CONCLUSÃO}

Com base na realização desse estudo, consideramos importante ressaltar o favorecimento para o crescimento pessoal e profissional como bolsista de iniciação científica. Embora ainda de maneira exploratória, pudemos obter mais conhecimento acerca do teste do reflexo vermelho com recém-nascidos e da avaliação ocular global.

Ao exame ocular externo, como observamos, a maioria dos recémnascidos estudados apresentaram algumas alterações comuns, principalmente edema palpebral, secreção ocular e estrabismo.

Quanto ao seguimento visual, 69 (45,4\%) dos recém-nascidos revelaram resposta classificada como intensamente reagente, portanto, com bom acompanhamento visual.

No referente ao resultado do teste do reflexo vermelho, $156(86,7 \%)$ mostraram resultado não alterado e 24 (13,3\%) suspeito. Após avaliação oftalmológica pelo especialista da instituição, estes apresentaram retinopatia da prematuridade, retina avascular e pontos hemorrágicos na retina. Os demais recém-nascidos com resultado suspeito do reflexo vermelho foram encaminhados para avaliação pela oftalmologista colaboradora desse estudo.

Ao considerarmos a relevância do estudo, na nossa opinião este sobressai não apenas para a promoção da saúde ocular do recém-nascido, mas também para a saúde global da criança. Afinal, o papel da visão no desenvolvimento físico e cognitivo normal é um fato inquestionável, visto que as pesquisas desenvolvidas por vários profissionais atuantes na área mostram quais as interferências da privação destas no dia-a-dia do ser humano. Ademais, experiências como esta podem fazer-nos despertar para a importância do trabalho do enfermeiro, sobretudo em conjunto com outros profissionais que cuidam diretamente da criança. Dessa forma, eles estarão atuando melhor ao inserir a prática do teste do reflexo vermelho na rotina de cuidados, com vistas a promover a prevenção de alterações visuais logo na infância.

Na nossa opinião, o desenvolvimento de pesquisas nessa área se faz pertinente, pois no mundo afora, e em especial no Brasil, a cegueira em crianças tem atingido índices significativos, representando sério problema de saúde pública ainda mais grave quando consideramos que a maioria dos casos poderiam ter sido prevenidos ou tratados pela adoção de medidas simples.

Portanto, é preciso se estimular tais pesquisas em busca de resultados mais promissores para a prevenção e redução da cegueira infantil.

\section{REFERÊNCIAS}

1. Costa KAB, Cardoso MVLML, Lúcio IML. Avaliação visual do recém-nascido no ambiente hospitalar. Rev Paul Enferm 2005; 24(2): 23-9.

2. Ventura LM, Travassos S, Marques S. Um programa baseado na triagem visual de recém-nascidos em maternidades. Fundação Altino Ventura/ 2002. Arq Bras Oftalmol 2002; 65(6): 629-35.

3. Gilbert C, Rahi J, Quinn G. Visual impairtment and blindess in children. In: Johnson G, Minassian B, Weale W, West S, editors. Epidemiology of eye disease. London (UK): Arnoud; 2003.

4. Margotto PR. Assistência ao recém-nascido de risco. In: Segre AM. Perinatologia, fundamentos, prática. $2^{\mathrm{a}}$ ed. São Paulo (SP): Sarvier; 2005.

5. Silva GRF. Estimulação visual: prática educativa com mães na enfermaria mãe-canguru (dissertação de mestrado). Fortaleza (CE): Universidade Federal do Ceará; 2005.

6. Dantas RA, Cardoso MVLML. Alterações oculares no escolar e a participação dos pais. Pediatria Moderna 2002; 38(11): 517-22.

7. Hugonnier-Clayette $S$, Magnard $P$, Bourron-Maidignier M, Hullo A. As deficiências visuais na infância: deficiências e adaptações.
São Paulo (SP): Manole; 1989.

8. Nettina SM. Prática de enfermagem. Rio de Janeiro (RJ): Guanabara Koogan; 1998.

9. Lúcio IML. Método de avaliação visual aplicado ao recém-nascido. (dissertação de mestrado). Fortaleza (CE): Universidade Federal do Ceará; 2004.

10. Reis PP. Reflexo vermelho. Textos científicos. Belo Horizonte (MG): Sociedade Mineira de Pediatria; 2005.

11. Lima AJ. Pediatria essencial. São Paulo (SP): Atheneu; 1992.

12. American Academy of Pediatrics. Eye examination in infants, children and young adults by pediatricians. Ophthalmology 2003; 110: 860-5.

13. Branden PS. Enfermagem materno-infantil. Rio de Janeiro (RJ): Reichmann \& Affonso; 2000.

14. Kenner C. Enfermagem neonatal. Rio de Janeiro (RJ): Reichmann \& Affonso; 2001.

15. Costa KAB, Cardoso MVLML. Exame do reflexo vermelho em recém-nascidos. Relatório de Pesquisa. PIBIC CNPq. Fortaleza (CE): Universidade Federal do Ceará; 2005. 